\title{
Animal model of personalized pharmacotherapy for alveolar bone regeneration
}

\section{Mariko Kawai, Kiyoshi Ohara}

\section{Department of Pharmacology, Osaka Dental University, Japan}

Background: The alveolar bone is very important for tooth retention. However, periodontitis or trauma cause loss of the alveolar bone, which is not spontaneously regenerated. Moreover, the alveolar bone is small size and it is critical to be regenerated only in the proper areas. Therefore, bone regeneration therapy for alveolar bone is necessary to be personalized properly. Methods: We made the electrodes for the alveolar bones of the first molar in the maxilla using dental impression system. We injected Bone Morphogenetic Protein (BMP)-2/7 gene expression plasmid vector into the periodontal tissues of the first molar in the maxilla of 9-weel-old male Wistar rats and immediately electroporated in the condition of 50V., 50msec., 32 pulses. In the histological analyses, we obtained time course samples and conducted hematoxylin - eosin staining and immunohistochemical staining. To investigate the mineral apposition rate (MAR) of alveolar bone in the target site, we performed double bone staining using calcein and tetracycline.

Results: Five days after the BMP-2/7 gene transfer, the new alveolar bones were formed in the target areas. Two weeks later, the additional alveolar bones appeared like as the mini-modeling. MAR of the target areas were rapid than that of the control site.

Conclusions: We constructed the animal model of the personalized pharmacotherapy for alveolar bone regeneration in rats. Therefore, it could be expected to be applied this system clinically. 